\title{
Acute respiratory distress syndrome
}

\author{
Authors: Christopher Mason, ${ }^{A}$ Nessa Dooley ${ }^{B}$ and Mark Griffiths ${ }^{C}$
}

Acute respiratory distress syndrome is a common cause of acute respiratory failure that is underdiagnosed both inside and outside of intensive care units. Progression to the most severe forms of the syndrome confers a mortality rate greater than $40 \%$ and is associated with often severe functional disability and psychological sequelae in survivors. While there are no disease-modifying pharmacotherapies for the syndrome, this progression may be prevented through the institution of quality improvement measures that minimise iatrogenic injury associated with acute severe illness.

KEYWORDS: Acute respiratory distress syndrome, acute respiratory failure, ARDS, critical illness, ventilator-associated lung injury

\section{Definitions, epidemiology and outcomes}

Acute respiratory distress syndrome (ARDS), at the time termed the adult respiratory distress syndrome, was first reported in a case series from Denver in 1967. ${ }^{1}$ Forty-five years later, the syndrome was 'relaunched', with the announcement of the 'Berlin' definition (Table 1). This iteration of the definition was validated using retrospective cohorts and captures patients with a mortality of $24 \%$ rising to $48 \%$ in the group of patients with the most severe respiratory failure. ${ }^{2}$

Using the previous, less robust, definition of ARDS, several population-based studies showed a fairly consistent picture of the age, mortality, and severity of illness; however, there is almost a fourfold difference in incidence, probably contributed to by differences in study design and intensive care unit (ICU) utilisation. ${ }^{3}$ In the USA, there are estimated to be 190,000 cases and 74,000 deaths annually from ARDS; ${ }^{4}$ whereas in a third world setting, of 1,046 patients admitted to a Rwandan referral hospital over 6 weeks, $4 \%$ (median age 37 years) met modified ARDS criteria, only $30.9 \%$ of patients with ARDS were admitted to an ICU and hospital mortality was 50.0\%. This study used the Kigali modification of the Berlin definition: without requirement for positive end-expiratory pressure, hypoxia threshold of $\mathrm{SpO}_{2} / \mathrm{FiO}_{2}$ less than or equal to 315, and bilateral opacities on lung ultrasound or chest radiograph. ${ }^{5}$

Authors: ${ }^{\text {A }}$ specialist registrar, Barts Heart Centre, St Bartholomew's Hospital, London, UK; ${ }^{\text {B }}$ specialist registrar, National Heart \& Lung

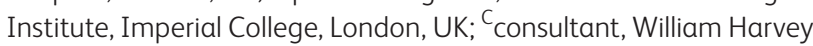
Research Institute, Queen Mary University of London, London, UK
The recently published Lung SAFE trial was designed to prospectively study the performance of the Berlin definition and to reflect modern management of ARDS. To those ends, the investigators recorded admissions over 4 weeks to 459 ICUs in 50 countries over 5 continents, totalling 29,144 patients. Of the admissions recorded, 3,022 (10.4\%) cases fulfilled ARDS criteria, including almost a quarter of those supported with invasive mechanical ventilation. ${ }^{6}$ Despite this relatively high prevalence and the study's focus on ARDS, the syndrome was recognised in only half of the mild ARDS group. Furthermore, in a study that reported on 815 patients with at least one risk factor for ARDS who were admitted to one of three Spanish hospitals over 4 months, 15 out of 53 patients (28\%) were not admitted to an ICU, suggesting that Lung SAFE may have both underestimated ARDS incidence and over-looked diagnoses. ${ }^{7}$

Survivors commonly suffer from muscle weakness and neuropsychiatric problems, such that fewer than $50 \%$ have returned to work 12 months after leaving intensive care. ${ }^{8}$ However, it is unusual for ARDS survivors to be significantly limited by chronic respiratory failure. Therefore, ARDS is important both clinically and financially, because it is a not uncommon contributor to the deaths of critically ill patients of all ages and because survivors carry on suffering from the after effects of critical illness long after they leave hospital. ${ }^{9}$ It is important to emphasise that, as a syndrome rather than a disease, there is no laboratory, imaging or other 'gold standard' investigation to diagnose ARDS. Like acute kidney injury, ARDS is caused by a huge range of conditions and, as a consequence, patients with ARDS are heterogeneous. Hence, the outcome for these patients is determined by the underlying causes of ARDS, patientspecific factors such as comorbidities, the way the illness is managed and the severity of illness.

\section{Pathophysiology}

Acute inflammation or injury at the lung's alveolar-capillary membrane increases the permeability of the membrane associated with the recruitment of neutrophils and other mediators of acute inflammation into the airspace. This gives rise to two processes that underlie the pathophysiology of ARDS. The resulting acute inflammatory exudate inactivates surfactant leading to collapse and consolidation of distal lung parenchyma with progressive loss of the lung's gas exchange surface area. This loss would be compensated for by hypoxic pulmonary vasoconstriction if the inflammatory process did not also effectively paralyse the lung's means of controlling vascular tone, thereby allowing deoxygenated blood to 


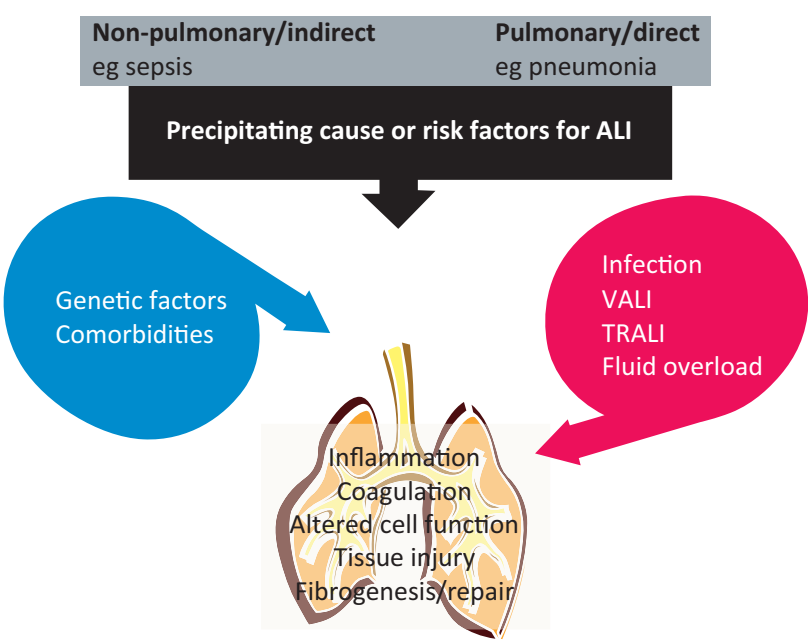

Fig 1. The evolution of acute respiratory distress syndrome (ARDS). The aetiology of the syndrome depends on one or more initiating factors that may affect the lung directly or via the circulation. These interact with patientspecific modulators (blue) and iatrogenic factors (red). The sum of a variety of pathological mechanisms manifests as ARDS. ALI = acute lung injury; TRALI = acute lung injury associated with the transfusion of blood products; VALI $=$ ventilator-associated lung injury

cross unventilated lung units on its way to the left heart. The combination of these two processes causes profound hypoxaemia associated with high permeability pulmonary oedema.

\section{ARDS prevention}

No drug treatment is known to improve the outcome of ARDS, despite decades of clinical trials. This has led to an interest in testing the ability of agents to either prevent ARDS or stop the progression from mild to more severe forms of the syndrome.

Research into critical illness has increased our understanding of the contribution of iatrogenic factors, most notably fluid overload, ventilator-associated lung injury (VALI) from mechanical ventilation, transfusion of blood products and hospital acquired infection (Fig 1), in ARDS. ${ }^{10}$ While it is sobering to appreciate the negative role that healthcare systems have played, it has at least indicated the potential to prevent ARDS through simple quality improvement interventions. ${ }^{11,12}$

The Lung Injury Prediction Score (LIPS; Table 1) is the product of a series of epidemiological studies. ${ }^{13,14}$ LIPS was designed to identify a population of patients at high risk of ARDS for prevention studies to be carried out by the National Institutes of Health's Prevention and Early Treatment of Acute Lung Injury (PETAL) Network (http://petalnet.org/). A score of greater than 4 points conferred sensitivity for acute lung injury (ALI) of 0.69 (95\% CI $0.64-0.74)$, specificity of 0.78 (95\% CI 0.77-0.79), positive predictive value $0.18(0.16-0.20)$ and negative predictive value 0.97 (0.97-0.98). LIPS-A is the first large multicentre study to address the question of whether ARDS can be prevented with a drug, in this case aspirin - the latest in a succession of promising therapeutics for ARDS, which was supported by a plethora of positive preclinical data and basic science. Disappointingly, the study was negative and

\begin{tabular}{|c|c|c|c|}
\hline $\begin{array}{l}\text { Predisposing } \\
\text { conditions }\end{array}$ & $\begin{array}{l}\text { LIPS } \\
\text { points }\end{array}$ & Risk modifiers & $\begin{array}{l}\text { LIPS } \\
\text { points }\end{array}$ \\
\hline Shock & 2 & Alcohol abuse & 1 \\
\hline Aspiration & 2 & Obesity (BMI>30) & 1 \\
\hline Sepsis & 1 & Hypoalbuminaemia & 1 \\
\hline Pneumonia & 1.5 & Diabetes mellitus & -1 \\
\hline High-risk surgery & & Chemotherapy & 1 \\
\hline$>$ Orthopaedic spine & 1 & $\mathrm{FiO}_{2}>0.35$ or $>4 \mathrm{~L} / \mathrm{min}$ & 2 \\
\hline > Acute abdomen & 2 & Tachypnoea RR>30 & 1.5 \\
\hline$>$ Cardiac & 2.5 & $\mathrm{SpO}_{2}<95 \%$ & 1 \\
\hline$>$ Aortic vascular & 3.5 & Acidosis ( $\mathrm{pH}<7.35)$ & 1.5 \\
\hline \multicolumn{4}{|l|}{ High-risk trauma } \\
\hline$>$ Traumatic brain injury & 2 & & \\
\hline$>$ Smoke inhalation & 2 & & \\
\hline$>$ Near drowning & 2 & & \\
\hline > Lung confusion & 1.5 & & \\
\hline > Multiple fractures & 1.5 & & \\
\hline \multicolumn{4}{|c|}{$\begin{array}{l}\text { In evaluation, LIPS discriminated between patients who did and who did } \\
\text { not develop acute lung injury (ALI). When a cut-off score of greater than } 4 \\
\text { points was used, sensitivity of the score for ALI was } 0.69 \text { ( } 95 \% \text { CI } 0.64-0.74 \text { ), } \\
\text { specificity } 0.78 \text { ( } 95 \% \text { CI } 0.77-0.79 \text { ), positive predictive value } 0.18(0.16-0.20) \\
\text { and negative predictive value } 0.97(0.97-0.98)^{13}\end{array}$} \\
\hline
\end{tabular}

one contributing factor was that the score threshold for study inclusion produced only half the predicted number of ARDS cases, the study's primary outcome. ${ }^{15}$ This does raise concerns about the ability of LIPS to identify an enriched population of patients at risk for ARDS without the addition of factors such as biomarkers that can predict deterioration from at risk to mild ARDS, severe ARDS and death.

Two further strategies have been employed to investigate ARDS prevention. Elective high-risk surgery - most commonly oesophagectomy, which confers a risk of post-operative ARDS of up to $20 \%$ - can be used as a model of ARDS in phase II clinical trials. Hence, the HARP investigators demonstrated proof of principle for simvastatin $(80 \mathrm{mg}$ before and after the operation for 11 days in total) in attenuating biochemical markers of lung injury, but not physiological indices, in oesophagectomy patients. ${ }^{16}$ Secondly, by characterising patients early in their clinical course, before they develop ARDS, it has been possible to refine the parameters to the need for supplemental oxygen, an elevated respiratory rate and bilateral infiltrates on the chest radiograph to identify patients with early acute lung injury (EALI). ${ }^{17}$ A prospective multicentre study is needed to evaluate the positive predictive value of an EALI score comprising these variables that could have a similar role to LIPS in future trials.

\section{ARDS management}

Despite advances in our understanding of the epidemiology and pathogenesis of ARDS, this has failed to lead to a single effective 'disease-modifying' therapy. ${ }^{18}$ Corticosteroids are ineffective 
at preventing the syndrome and numerous studies have failed to determine whether their use is beneficial in patients who fail to improve after the first week of ARDS. Many supposed experts, ourselves included, use methylprednisolone (for example, $2 \mathrm{mg} / \mathrm{kg}$ per day in divided doses for 2 weeks, followed by $1 \mathrm{mg} / \mathrm{kg}$ for 2 more weeks) in patients who 'get stuck'. However, the essence of management of these cases consists of optimising diagnosis and treatment of underlying conditions, and the deployment of supportive measures that minimise iatrogenic injury and the consequences of severe critical illness (ie secondary and tertiary prevention).

\section{Diagnosis}

Any diagnostic strategy for ARDS is sufficiently dependent on local factors, such as the prevalent causes of infectious pneumonia and access to imaging modalities (like transoesophageal echocardiography), that a single protocol cannot be recommended. Similarly, local guidelines should be regularly updated. There are two main differential diagnoses (conditions that resemble ARDS but have a distinct pathophysiology):

1 cardiovascular conditions of rapid onset, including left heart failure, right-to-left vascular shunts, usually with some lung pathology, and major pulmonary embolism

2 lung conditions that develop more slowly than ARDS, for example interstitial lung diseases (especially acute interstitial pneumonia), broncho-alveolar cell carcinoma, lymphangitis and the pulmonary vasculitides.

\section{Secondary and tertiary prevention}

According to the available evidence, the central tenant of management of ARDS is the prevention and mitigation of VALI, which is discussed in the next section.

Maintenance of a neutral fluid balance was associated with decreased length of ICU stay and ventilator dependency without evidence of increased non-pulmonary organ failure in the ARDS Network Fluid and Catheter Treatment Trial (FACTT). ${ }^{19}$ Strategies that may be used to achieve a negative fluid balance include intake restriction, diuresis and haemofiltration. Considerable judgement is required to determine how negative a fluid balance to attempt and when in the clinical course the patient's microvasculature is no longer 'leaky', such that fluid balance restrictions can be relaxed. As a note of caution, one follow-up study from FACTT has demonstrated an association between conservative fluid balance strategy and cognitive impairment in survivors. ${ }^{20}$

Transfusion of blood products has been associated with the incidence of ARDS, nosocomial infection and mortality in critical illness. Transfusion-related lung injury (TRALI) is defined as the onset of ARDS within 6 hours of the transfusion of any blood product in the absence of another risk factor. ${ }^{21}$ The most important mechanism of injury appears to be the interaction of pre-formed antibodies in the product with the host pulmonary vascular endothelium. Hence, products containing the most plasma confer the highest risk and the exclusion of female donors of products with high plasma volume has resulted in a decrease in the incidence of TRALI by roughly two thirds. Transfusion of packed red cells using a threshold of seven was non-inferior to a threshold of $9 \mathrm{~g} / \mathrm{dL}$; corresponding protocols restricting unnecessary transfusion should be introduced locally and practices audited.

There is a dearth of evidence-based practices that decrease hospital acquired infection. An effective local antibiotic policy should aim to optimise antibiotic treatment according to local surveillance data and to ensure rapid de-escalation based on culture results. Recent evidence suggests that enteral nutrition is preferable to parenteral, and that under feeding is less dangerous than over provision. Finally, active rehabilitation, specialist outpatient follow up and psychological support have been recommended for all survivors of severe critical illness in order to mitigate the neuro-psychological effects and weakness. $^{22}$

\section{Ventilatory management and the avoidance of ventilator-associated lung injury}

Arguably all of the strategies that improve outcome in ARDS have their effect by preventing VALI. ${ }^{23}$ Low tidal volume ventilation is the mainstay of management. The ARMA ARDS Network study demonstrated a significant mortality advantage associated with a ventilation strategy targeting a tidal volume of $6 \mathrm{~mL} / \mathrm{kg}$ predicted body weight and a plateau pressure $<30 \mathrm{cmH}_{2} 0$ in 861 patients. However, reanalysis of the data suggests that achieving an even lower tidal volume may confer additional benefit, which in part underlies the use of the extracorporeal lung support techniques of carbon dioxide removal (ECCOR) and extracorporeal membrane oxygenation (ECMO). While a robust evidence base for the use of the former in ARDS is currently lacking, referral of patients with potentially reversible, severe ARDS to a regional ECMO centre improved mortality and was cost effective. ${ }^{24}$ After the H1N1 2009 influenza pandemic in which the use of ECMO appeared to confer benefit, ${ }^{25}$ a network of five ECMO centres was commissioned to provide advanced care for patients with severe acute respiratory failure in England. Rescue therapies for refractory hypoxaemia that may be used for patients who are unsuitable for ECMO or to temporise include recruitment manoeuvres, alternative ventilation modes and inhaled vasodilators (eg nitric oxide and prostacyclin). These strategies do not improve the outcome of unselected patients with ARDS; indeed, the use of high frequency oscillation ventilation was associated with an increased mortality despite short-term improvement in gas exchange. ${ }^{26,27}$

An individual patient data meta-analysis of three trials examining the role of high versus lower levels of positive end-expiratory pressure in patients with ARDS suggested that patients with moderate or severe ARDS may benefit from the application of higher levels. ${ }^{28}$ Prone positioning improves oxygenation in approximately two thirds of ARDS patients by improving ventilation/perfusion matching, and may improve mortality by sharing VALI between the anterior and posterior portions of the lung. Available evidence suggests that the observed survival benefit is restricted to patients with moderate to severe ARDS, who are placed in the prone position for at least 16 hours per day. ${ }^{29}$ Similarly, a multicentre randomised controlled trial evaluating the use of 48 hours neuromuscular blockade with cisatracurium in patients with moderate to severe ARDS $\left(\mathrm{PaO}_{2} / \mathrm{FiO}_{2}\right.$ ratio $\left.<150 \mathrm{~mm} \mathrm{Hg}\right)$ improved 
mortality and increased ventilator and organ failure free days without any difference in ICU acquired paresis. ${ }^{30}$

\section{Future directions}

There are no disease modifying drug therapies for ARDS. Drug development in this area is notoriously difficult, partly because ARDS is not a disease but a label describing acute respiratory failure occurring de novo as a result of a wide variety of conditions. One strategy designed to increase the likelihood of positive clinical trials in ARDS is to select a less heterogeneous patient population - a step on the road to a personalised approach made at the expense of having a smaller pool of patients from which to recruit. Such splitting can be envisaged on the basis of readily identifiable predisposing causes (eg influenza pneumonia, transfusion-related acute lung injury or systemic sepsis) or inherent patient characteristics, such as alcoholism or the expression of particular single nucleotide polymorphisms known to be associated with a predisposition to ARDS (eg the functional promoter variants in sphingosine 1-phosphate receptor 3). ${ }^{31}$ The ultimate aim is to identify subgroups, so-called endotypes, of ARDS that will predict a positive response to a certain class of therapy. ${ }^{32}$

\section{Conclusions}

Current management of ARDS is hampered by failure to diagnose the condition and to prevent iatrogenic harms. We need to heighten awareness of the diagnosis, particularly outside ICUs, so that the opportunity to prevent progression of the syndrome is not missed. Finally, research into prevention and treatment needs to be translated more effectively into the clinic.

In order to discover effective drug therapies, we need to continue to invest in human studies that aim to elucidate the pathogenesis of ARDS and to identify clinically useful biomarkers and surrogate outcome measures. ${ }^{33,34}$ These investigations need to be performed with a view to designing a step-wise approach to testing novel therapeutics in this particularly challenging patient group. Finally, phenotyping patients with a view to predicting drug class response may further help to establish disease modifying therapies.

\section{Conflicts of interest}

MG has received grants, personal fees and non-financial support from GSK, grants from Pfizer, and personal fees from BI, Peptinnovate, Silence Therapeutics and Cell Catapult.

\section{References}

1 Ashbaugh DG, Bigelow DB, Petty TL, Levine BE. Acute respiratory distress in adults. Lancet 1967;2:319-23.

2 Ferguson ND, Fan E, Camporota L et al. The Berlin definition of ARDS: an expanded rationale, justification, and supplementary material. Intensive Care Med 2012;38:1573-82.

3 Rubenfeld GD, Herridge MS. Epidemiology and outcomes of acute lung injury. Chest 2007;131:554-62.

4 Rubenfeld GD, Caldwell E, Peabody E et al. Incidence and outcomes of acute lung injury. N Engl J Med 2005;353:1685-93.

5 Riviello ED, Kiviri W, Twagirumugabe T et al. Hospital incidence and outcomes of the acute respiratory distress syndrome using the Kigali modification of the Berlin definition. Am J Respir Crit Care Med 2016;193:52-9.
6 Bellani G, Laffey JG, Pham T et al. Epidemiology, patterns of care, and mortality for patients with acute respiratory distress syndrome in intensive care units in 50 countries. JAMA 2016;315:788-800.

7 Ferguson ND, Frutos-Vivar Esteban A et al. Clinical risk conditions for acute lung injury in the intensive care unit and hospital ward: a prospective observational study. Crit Care 2007;11:R96.

8 Herridge MS, Cheung AM, Tansey CM et al. One-year outcomes in survivors of the acute respiratory distress syndrome. $\mathrm{N} \mathrm{Engl} \mathrm{J} \mathrm{Med}$ 2003;348:683-93.

9 Herridge MS, Tansey CM, Matté A et al. Functional disability 5 years after acute respiratory distress syndrome. $N$ Engl J Med 2011;364:1293-304.

10 Salman D, Finney SJ, Griffiths MJ. Strategies to reduce ventilatorassociated lung injury (VALI). Burns 2013;39:200-11.

11 Herasevich V, Tsapenko M, Koijicic M et al. Limiting ventilatorinduced lung injury through individual electronic medical record surveillance. Crit Care Med 2011;39:34-9.

12 Pickering BW, Litell JM, Herasevich V, Gajic O. Clinical review: the hospital of the future - building intelligent environments to facilitate safe and effective acute care delivery. Crit Care 2012;16:220.

13 Gajic $\mathrm{O}$ et al. Early identification of patients at risk of acute lung injury: evaluation of lung injury prediction score in a multicenter cohort study. Am J Respir Crit Care Med 2011;183:462-70.

14 Trillo-Alvarez C, Cartlin-Ceba R, Kor DJ et al. Acute lung injury prediction score: derivation and validation in a population-based sample. Eur Respir J 2011;37:604-9.

15 Kor DJ, Carter RE, Park PK et al. Effect of aspirin on development of ARDS in at-risk patients presenting to the emergency department: the LIPS-A randomized clinical trial. JAMA 2016;315:2406-14.

16 Shyamsundar M, McAuley DF, Shields MO et al. Effect of simvastatin on physiological and biological outcomes in patients undergoing esophagectomy: a randomized placebo-controlled trial. Ann Surg 2014;259:26-31.

17 Rackley CR, Levitt JE, Zhuo H, Matthay MA, Calfee CS. Clinical evidence of early acute lung injury often precedes the diagnosis of ALI. J Intensive Care Med 2013;28:241-6.

18 Boyle AJ, Mac Sweeney R, McAuley DF. Pharmacological treatments in ARDS; a state-of-the-art update. BMC Med 2013;11:166.

19 National Heart, Lung, and Blood Institute Acute Respiratory Distress Syndrome (ARDS) Clinical Trials Network. Comparison of two fluid-management strategies in acute lung injury. $\mathrm{N} \mathrm{Engl} \mathrm{J} \mathrm{Med}$ 2006;354:2564-75.

20 Mikkelsen ME, Christie JD, Lanken PN et al. The adult respiratory distress syndrome cognitive outcomes study: long-term neuropsychological function in survivors of acute lung injury. Am J Respir Crit Care Med 2012;185:1307-15.

21 Vlaar AP, Juffermans NP. Transfusion-related acute lung injury: a clinical review. Lancet 2013;382:984-94.

22 National Institute for Health and Care Excellence. Rehabilitation after critical illness in adults. NICE clinical guideline No 83. Manchester: NICE, 2009.

23 Slutsky AS, Ranieri VM. Ventilator-induced lung injury. $N$ Engl $J$ Med 2013;369:2126-36.

24 Peek GJ, Mugford M, Tiruvoipati R et al. Efficacy and economic assessment of conventional ventilatory support versus extracorporeal membrane oxygenation for severe adult respiratory failure (CESAR): a multicentre randomised controlled trial. Lancet 2009;374;1351-63.

25 Noah MA, Peek GJ, Finney SJ et al. Referral to an extracorporeal membrane oxygenation center and mortality among patients with severe 2009 influenza A(H1N1). JAMA 2011;306:1659-68.

26 Ferguson ND, Cook DJ, Guyatt GH et al. High-frequency oscillation in early acute respiratory distress syndrome. $N$ Engl J Med 2013;368:795-805.

27 Young D, Lamb SE, Shah S et al. High-frequency oscillation for acute respiratory distress syndrome. N Engl J Med 2013;368:806-13. 
28 Briel M, Meade M, Mercat A et al. Higher vs lower positive endexpiratory pressure in patients with acute lung injury and acute respiratory distress syndrome: systematic review and meta-analysis. JAMA 2010;303:865-73.

29 Guèrin C, Reignier J, Richard JC et al. Prone positioning in severe acute respiratory distress syndrome. N Engl J Med 2013;368:2159-68.

30 Papazian L, Forel JM, Gacouin A et al. Neuromuscular blockers in early acute respiratory distress syndrome. $N$ Engl J Med 2010;363:1107-16.

31 Sun X, Ma SF, Wade MS et al. Functional promoter variants in sphingosine 1-phosphate receptor 3 associate with susceptibility to sepsis-associated acute respiratory distress syndrome. Am J Physiol Lung Cell Moll Physiol 2013;305:L467-77.
32 Prescott HC, Calfee CS, Thompson BT, Angus DC, Liu VX. Towards smarter lumping and smarter splitting: rethinking strategies for sepsis and ards clinical trial design. Am J Respir Crit Care Med 2016;194:147-55.

33 Proudfoot AG, Hind M, Griffiths MJ. Biomarkers of acute lung injury: worth their salt? BMC Med 2011;9:132.

34 Proudfoot AG, McAuley DF, Griffiths MJ, Hind M. Human models of acute lung injury. Dis Model Mech 2011;4:145-53.

Address for correspondence: Dr M Griffiths, Office 10 (BNB_01_411), 1st Floor - KGV Building, St Bartholomew's Hospital, West Smithfield, London EC1A 7BE, UK.

Email: m.griffiths@ic.ac.uk 